\title{
The Weyl tensor in Spatially Homogeneous Cosmological Models
}

\author{
John D. Barrow* and Sigbjørn Hervik ${ }^{\dagger}$ \\ DAMTP, \\ Centre for Mathematical Sciences, \\ Cambridge University, \\ Wilberforce Rd., \\ Cambridge CB3 0WA, UK
}

April 1, 2018

\begin{abstract}
We study the evolution of the Weyl curvature invariant in all spatially homogeneous universe models containing a non-tilted $\gamma$-law perfect fluid. We investigate all the Bianchi and Thurston type universe models and calculate the asymptotic evolution of Weyl curvature invariant for generic solutions to the Einstein field equations. The influence of compact topology on Bianchi types with hyperbolic space sections is also considered. Special emphasis is placed on the late-time behaviour where several interesting properties of the Weyl curvature invariant occur. The late-time behaviour is classified into five distinctive categories. It is found that for a large class of models, the generic late-time behaviour the Weyl curvature invariant is to dominate the Ricci invariant at late times. This behaviour occurs in universe models which have future attractors that are planewave spacetimes, for which all scalar curvature invariants vanish. The overall behaviour of the Weyl curvature invariant is discussed in relation to the proposal that some function of the Weyl tensor or its invariants should play the role of a gravitational 'entropy' for cosmological evolution. In particular, it is found that for all ever-expanding models the measure of gravitational entropy proposed by Grøn and Hervik increases at late times.
\end{abstract}

\section{Introduction}

What is the entropy of a gravitational field? Following the insights of Hawking and Bekenstein [1. 2], an answer has been only been provided for a subset of situations characterised by static and stationary spacetimes with event horizons. In particular, black holes are found to be black bodies. They possess a well-defined entropy. They obey the laws of equilibrium thermodynamics and,

\footnotetext{
*J.D.Barrow@damtp.cam.ac.uk

†S.Hervik@damtp.cam.ac.uk
} 
when perturbed, their fluctuations obey the laws of close-to-equilibrium thermodynamics [3]. Yet, despite these developments it remains an unsolved problem whether an analogous gravitational entropy can be defined for non-stationary spacetimes of the sort that characterise most relativistic cosmologies that are considered as models for the past, present, and future of our visible universe. Unfortunately, a solution of this problem appears to involve the solution of a number of separate hard problems in gravitation physics. It requires a rigorous measure of the probability of different cosmological initial conditions to be found. It also requires us to be sure that we have accounted for all the possible contributions to the gravitational entropy: there may be quantum, classical, geometrical, topological, thermal, and dynamical contributions that need to be quantified and added [4. The nature of the states of zero and maximum entropy also need to be defined and the influence of possible events like inflation must be fully accounted for entropically, and all must be suitably coordinate covariant. It has also been argued [5, 6] that the whole concept of the arrow of time and thermodynamics relies upon the concept of a gravitational entropy, and hence leads all the way back to fundamental questions about the origin of time and the nature of the initial state of the universe.

Gravitation has a tendency to amplify small inhomogeneities in the distribution of matter. Yet, our present observations of the spectrum and temperature isotropy of the microwave background radiation show that the radiation was very close to thermal equilibrium when it was last-scattered, and that fluctuations from the Friedmann-Robertson-Walker (FRW) spacetime metric are only of order $10^{-5}$. If there does exist a gravitational entropy that grows with time to reflect the action of gravitational instability then it should have the property that it vanishes (or at least is small) for a homogeneous and isotropic metric and be maximal for a black hole of the same total mass. This type of observation has led some investigators to propose that any measure of cosmological gravitational entropy should be proportional to the deviation of the universe from the FRW model [7]. Hence, the present gravitational entropy of the universe is in some sense small and so must have been even smaller in the past, perhaps providing some rationale for assuming the initial state of the universe to have zero entropy and hence be FRW up to minimal quantum gravitational fluctuations.

Based on this observation, Penrose [8] suggested that the Weyl curvature invariant, given by

$$
C^{\alpha \beta \gamma \delta} C_{\alpha \beta \gamma \delta}=R^{\alpha \beta \gamma \delta} R_{\alpha \beta \gamma \delta}-2 R^{\alpha \beta} R_{\alpha \beta}+\frac{1}{3} R^{2},
$$

should be very small near the initial singularity, and then grow thereafter behaving as a proper measure of the gravitational entropy. This in all its editions, is what we will call the Weyl Curvature Conjecture (WCC). Wainwright and Anderson [9] had a different version of the WCC. They expressed the conjecture in terms of the relative magnitude of the Weyl and Ricci curvature invariants:

$$
P^{2}=\frac{C^{\alpha \beta \gamma \delta} C_{\alpha \beta \gamma \delta}}{R^{\mu \nu} R_{\mu \nu}} .
$$

However, based on observations from two different universe models and taking into account that the entropy should scale with the volume, Grøn and Hervik [10], [11] suggested that a better measure of a gravitational entropy would be

$$
\mathcal{S}=\sqrt{|g|} P .
$$


Most other studies of the WCC [12, 13, 14, 15, 16, 17, 18] use the square of the Weyl scalar, $C^{\alpha \beta \gamma \delta} C_{\alpha \beta \gamma \delta}$, or $P$, as the putative gravitational entropy for cosmological models. However, these candidates do not scale as the volume and so they do not capture correctly the dependence of a Weyl entropy on the volume.

We introduce the expansion-normalised shear:

$$
\Sigma=\frac{3}{2} \frac{\sigma_{\mu \nu} \sigma^{\mu \nu}}{\theta^{2}} .
$$

The shear tensor is a symmetric and trace-free tensor defined by

$$
\sigma_{\mu \nu}=\theta_{\mu \nu}-\frac{1}{3} \theta\left(g_{\mu \nu}+u_{\mu} u_{\nu}\right) .
$$

Here, $u_{\mu}$ is the normalised four-velocity of the comoving fluid; $\theta_{\mu \nu} \equiv u_{\mu ; \nu}$ is the expansion tensor of the comoving fluid and $\theta=\theta_{\mu}^{\mu}$ the volume expansion rate. The shear tensor tells us how anisotropic the universe is; in particular, $\Sigma=0$ if and only if the universe is isotropic. From the generalised Friedmann equation $(8 \pi G=c=1)$

$$
\frac{1}{3} \theta^{2}=\frac{1}{2} \sigma^{\mu \nu} \sigma_{\mu \nu}-\frac{1}{2}{ }^{(3)} R+\rho+\Lambda .
$$

Since the density, $\rho$, is positive, $\Sigma$ will be bounded by

$$
0 \leq \Sigma \leq 1
$$

whenever the spatial Ricci-scalar is non-positive: ${ }^{(3)} R \leq 0$. This inequality will be fulfilled in all of the homogeneous models, except in the type IX and the Kantowski-Sachs closed universes.

One of the aims of this paper will be to investigate the quantity $\mathcal{S}$ for all homogeneous models, and determine whether or not it has the appropriate behaviour for a gravitational entropy. Hence, we will try to determine whether or not $\mathcal{S}$ increases during the evolution of all homogeneous universes. We will also investigate the quantity $P$, because this quantity tells us something of the physical properties of the universe model under consideration and the general properties of the evolution of anisotropic universes. We will discuss, unless stated otherwise, only spatially homogeneous cosmologies with a non-tilted $\gamma$ law perfect fluid. We also assume that the matter sources obey the strong energy condition; the effects from inflationary fluids are considered elsewhere [11]. We will investigate all the possible spatially homogeneous models. Of special interest are the eight Thurston geometries [19] which are related to the classification of topologies in three dimensions [20]. These models correspond to the Bianchi types in a rather interesting way 21, 22, 23. The possibility of compactification of these Thurston models has induced a renewed interest in the homogeneous models of Thurston and Bianchi type [24, 25, 26, 27].

This paper is organised as follows. In section 2 we investigate all the models that have the FRW models as a special case. The remaining Thurston models are investigated in section 3, and in section 4 we investigate the remaining Bianchi models of non-Thurston type. We summarise our results in the final section. 


\section{Models with maximally symmetric Thurston geometries}

First, we will consider models which contain the FRW universes as special cases. The spatial sections in these cases are either $\mathbb{E}^{3}, \mathbb{H}^{3}$, or $S^{3}$. In the isotropic case these correspond to the Bianchi types I and $\mathrm{VII}_{0}, \mathrm{~V}$ and $\mathrm{VII}_{h}$, and IX, which contain the $k=0,-1$, and +1 FRW universes respectively.

\subsection{The case $\mathbb{E}^{3}$}

For the flat case, ${ }^{(3)} R=0$, we have already exhaustively examined the Bianchi type I models in earlier papers 10, 11. An important class of solutions is provided by the Kasner vacuum solutions:

$$
d s^{2}=-d t^{2}+t^{2 p_{1}} d x^{2}+t^{2 p_{2}} d y^{2}+t^{2 p_{3}} d z^{2}
$$

The exponents obey $\sum_{i} p_{i}=\sum_{i} p_{i}^{2}=1$. The solution space of these solutions is a circle, and are usually referred to as the Kasner circle. Iff $p_{1}=1$, then the line element is Taub's form of flat spacetime. This form of flat spacetime will be referred to as $T$, and lies on the Kasner circle. Note that $T$ is one of two Locally Rotationally Symmetric (LRS) spacetimes on the Kasner circle. All vacuum Kasner solutions have maximal expansion-normalised shear:

$$
\Sigma=1 .
$$

We will assume a perfect fluid with equation of state linking the pressure $p$ and density $\rho$ :

$$
p=(\gamma-1) \rho .
$$

For all $\gamma<2$ it can be shown that the Kasner circle is a past attractorf Calculating the Weyl tensor, we find that near the initial singularity

$$
\left(C^{\alpha \beta \gamma \delta} C_{\alpha \beta \gamma \delta}\right)_{I} \propto t^{-4}
$$

where $t$ is the cosmological time. The quantities $P$ and $\mathcal{S}$ defined in eqs. (2) and (3), can be shown to behave as (assuming a Kasner-like behaviour)

$$
\begin{aligned}
& P_{I} \propto t^{\gamma-2} \\
& \mathcal{S}_{I} \propto t^{\gamma-1}
\end{aligned}
$$

to leading order near the initial singularity. We note that $P$ will diverge near the initial singularity for all $\gamma<2 ; \mathcal{S}$ will approach zero for $\gamma>1$ and diverge for $\gamma<1$. If a fluid with $\gamma \geq 1$ is assumed to dominate near the initial singularity then $\mathcal{S}$ will increase as $t \rightarrow 0$. Radiation fluids will dominate the early history of our universe so long as particle interaction and scattering times are shorter than the expansion time. This may not be the case for asymptotically-free interactions between $10^{14}-10^{19} \mathrm{GeV}$ but is likely to be restored (subject to

\footnotetext{
${ }^{1}$ The metric has permutation symmetry [28], so any of the other exponents could equally well be 1 .

${ }^{2}$ This is because the shear invariant $\sigma^{\mu \nu} \sigma_{\mu \nu}$, can be viewed as a $\gamma=2$ fluid in the Friedmann equation (4). Hence, if the model contains a perfect fluid with $\gamma<2$, the shear will dominate the initial singularity. This need not to be the case when $\gamma=2$.
} 
boundary effects on the long-wavelength part of the momentum distribution due to finite horizon effects and the breakdown of statistical mechanics) on approach to the Planck scale. Also, it is most likely that any self-interacting scalar fields present will become dominated by their kinetic energies as $t \rightarrow 0$. This is equivalent to domination by a $\gamma=2$ fluid. Thus an effective $\gamma>1$ in the neighbourhood of the initial singularity, is quite probable. It might not be the case if the potential energy of a scalar field dominated or scaled in proportion to its kinetic energy as $t \rightarrow 0$. An example of the latter type is provided by a scalar field $\varphi$ with an exponential potential $V(\varphi)=V_{0} \exp [-\lambda \varphi]$.

We see that for the point $T$ on the Kasner circle, we have

$$
\begin{aligned}
\left(C^{\alpha \beta \gamma \delta} C_{\alpha \beta \gamma \delta}\right)_{T} & \propto t^{-4} \cdot t^{2(2-\gamma)} \\
P_{T} & \propto \text { constant } \\
\mathcal{S}_{T} & \propto t
\end{aligned}
$$

near the initial singularity.

When we include a matter source with anisotropic pressure created by photons or asymptotically-free particles in an anisotropic universe (since they are expected to be collisionless significantly below the Planck temperature) or a cosmic magnetic field, the behaviour near the initial singularity turns out to be quite complex. The behaviour of a Bianchi type I model with a magnetic field [29] or Yang-Mills field [30] is chaotic. Similar behaviour are found in Bianchi type IX and VIII. In the types VIII and IX, curvature effects cause the universe to "bounce" between different Kasner states; the magnetic field in the type I case, tends to mimic this effect. The Bianchi type I with pure magnetic field was investigated using the dynamical systems approach in [29]. When the universe is in a Kasner epoch, the Weyl curvature invariant behaves as in the Kasner universe. The question to consider is: what happens to the Weyl curvature invariants during the bounces?

As we approach the initial singularity, the duration of the bounces will become negligible compared to the duration of the Kasner epochs in between the bounces. We can write the Weyl curvature invariant as [31]

$$
C^{\alpha \beta \gamma \delta} C_{\alpha \beta \gamma \delta}=H^{4} \mathcal{W}^{2}
$$

where $\mathcal{W}$ is the dimensionless Weyl curvature invariant, and $H=\theta / 3$ is the Hubble factor. The quantity $\mathcal{W}^{2}$ can be written so that it is only a polynomial (of degree 4) in the state space variables. The state space for Bianchi type I with a magnetic field is compact, and hence, all the variables are bounded and $\mathcal{W}^{2}$ must therefore also be bounded at all times. Similarly,

$$
R^{\mu \nu} R_{\mu \nu}=H^{4} \mathcal{R}^{2}
$$

where $\mathcal{R}^{2}$ is a positive definite polynomial of the bounded state-space variables. When the universe is in a Kasner epoch, $\mathcal{R}$ will vary as $\beta$

$$
\mathcal{R}_{I}^{2}=\mathcal{O}\left(t^{2(2-\gamma)}\right)+\mathcal{O}\left(t^{\frac{4}{3}(1-2 \cos \phi)}\right)
$$

\footnotetext{
${ }^{3}$ When an expression like this contains two terms, we should only consider the term which is the dominant one in each situation. Sometimes the real expression contains cross-terms which will lie in-between the two. These terms will be omitted in this paper, mainly for brevity.
} 
where $\phi$ is an angular variable on the Kasner circle. The first mode $t^{2(2-\gamma)}$ comes from the perfect fluid term, while $t^{\frac{4}{3}(1-2 \cos \phi)}$ comes from the energy density of the magnetic field. The behaviour of $P$ during these periods will therefore vary according to the value of the Kasner parameter $\phi$. The quantity $\mathcal{S}$ will behave as

$$
\mathcal{S}_{I}=\mathcal{O}\left(t^{\gamma-1}\right)+\mathcal{O}\left(t^{\frac{1}{3}(1+4 \cos \phi)}\right)
$$

during the Kasner epochs. If $\gamma>1$ this quantity will be increasing during this period. If $\gamma<1$ there will be Kasner epochs where $\mathcal{S}$ will decrease, and others where it will increase. On "average", assuming that all the Kasner epochs occur with equal probability, the quantity $\mathcal{S}$ will increase. During a bounce, the magnetic field will experience a sharp peak in its energy density. This makes $\mathcal{R}$ experience a peak as well. Hence, during this bounce, the quantity $\mathcal{S}$ will fall for a brief period, until it rises to a new Kasner level again. Thus, during Kasner oscillations, $\mathcal{S}$ will both decrease and increase in time. However, on average, $\mathcal{S}$ will increase continuously during these oscillations. If

The Bianchi type $\mathrm{VII}_{0}$ universe model, the other Bianchi model which is compatible with $\mathbb{E}^{3}$ spatial sections, has further interesting properties. For example, if $1 \leq \gamma \leq 4 / 3$ the solutions of this model will at late times isotropise in terms of the shear, but not isotropise with respect to the Weyl tensor [32, 33]. It can be shown that if $\gamma \leq 4 / 3$

$$
\Sigma_{V I I_{0}} \longrightarrow 0
$$

while for $\gamma>4 / 3$ the shear will oscillate as $t \longrightarrow \infty$ 34]. The Weyl tensor has a quite complicated behaviour; if $\gamma>1$ then $\mathcal{W}$ will oscillate with larger and larger amplitudes. The oscillations will diverge at late times in the sense that ${ }^{5}$

$$
\limsup _{t \longrightarrow \infty}\left|\mathcal{W}^{2}\right|=\infty
$$

This happens because the universe at late times will oscillate around an isotropic state. As the universe expands, the amplitude of the oscillations will be smaller and smaller, and hence, will isotropise with respect to the shear. But the oscillations will become more and more rapid while $\mathcal{W}$ increases beyond bound [32, 33].

The scalar $\mathcal{W}$ measures the absolute value of Weyl curvature, while the quantity $P$ measure the ratio of the "matter curvature" to the Weyl curvature. Strictly speaking, the measure $P$ will oscillate as the quantity $\mathcal{W}$ does, but we can avoid this by either adopting the definition of the Weyl parameter as in [32. 33], or we can take the absolute value of $\mathcal{W}^{2}$ and thereafter average over a complete period. We will do the latter, and denote these entities with a bar, for example $\bar{P}$. In the case of $\mathrm{VII}_{0}$ we then get

$$
\bar{P}_{V I I_{0}} \longrightarrow \infty, \quad \text { as } t \longrightarrow \infty
$$

So in this case, even the " $P$-version" of the WCC holds. Since the universe is ever expanding, $\mathcal{S}$ will diverge at late times as well, as

$$
\overline{\mathcal{S}}_{V I I_{0}} \longrightarrow \infty, \text { as } t \longrightarrow \infty \text {. }
$$

\footnotetext{
${ }^{4}$ Note that in physically realistic universes, which begin their cosmic evolution at the Planck time, there is time for very few oscillations to occur in the universe's history.

${ }^{5}$ The authors in [32, 33 define a "Weyl parameter" $\mathcal{W}$ differently from that defined here. This makes their parameter increase monotonically at late times, while ours will oscillate.
} 


\subsection{The hyperbolic spaces $\mathbb{H}^{3}$}

We will now consider the $\mathbb{H}^{3}$ cases. They have ${ }^{(3)} R<0$. The future asymptotics for these models are more easily obtained because they approach vacuum solutions in the future. All of the type $\mathrm{V}$ and $\mathrm{VII}_{h \neq 0}$ solutions will, assuming that the matter content obeys the strong energy condition, evolve asymptotically towards plane-wave spacetimes [35]. For plane waves, all the curvature scalars are zero even though the metrics are anisotropic. In general, these open universe solutions will not have a zero Weyl tensor (except for a set of zero measure which correspond to the isotropic vacuum Milne universes). However, the late-time behaviour of $\mathrm{VII}_{h}$ models is to approach particular exact solutions first found by Lukash [36] which are plane waves [35, 34. 5 .

This behaviour indicates that the evolution of the Weyl scalar and $\mathcal{S}$ in this case is more subtle as we approach the future asymptote. The Weyl scalars will tend to zero even though the anisotropy freezes in with $\Sigma$ approaching a constant value. Clearly, the Weyl scalar cannot capture the anisotropic plane-wave modes of the gravitational field. This can be considered as an objection to the argument that it is some function of the Weyl scalar which is the proper measure for the entropy. We need a more discriminating measure for the gravitational entropy, if it exists. It might also happen that these plane-wave spacetimes are isentropic modes corresponding to adiabatic perturbations of the gravitational field but we would need to know whether they are extendible into hermitian modes of a quantum cosmology.

In the $\mathrm{V}$ and $\mathrm{VII}_{h}$ cases containing a perfect fluid, part of the Kasner circle will be a past attractor for all equation of state parameters $\gamma<2$. Hence, the discussion for the type I model near the initial singularity also applies to these models.

As we already have mentioned, the future attractor is a Lukash plane-wave solution with $\Sigma \neq 0$ if $2 / 3<\gamma<2$ and the open FRW model with $\Sigma=0$ if $\gamma \leq 2 / 3$. Using the exact solutions in the type $\mathrm{V}$ model with perfect fluid (see [31]), we can show that when $2 / 3 \leq \gamma \leq 2$ the late-time behaviour for these solutions has a Weyl curvature invariant evolution

$$
\left(C^{\alpha \beta \gamma \delta} C_{\alpha \beta \gamma \delta}\right)_{V}=t^{-4} \cdot\left[\mathcal{O}\left(t^{-8}\right)+\mathcal{O}\left(t^{-3 \gamma-2}\right)\right] .
$$

The first of these terms comes from pure shear term effects in the absence of matter. The second term is driven by the matter interactions. In the presence of a perfect fluid, the pure shear term will always be sub-dominant for $2 / 3 \leq \gamma<2$. Under the assumption $2 / 3 \leq \gamma \leq 2$ at late times we have

$$
\begin{aligned}
& P_{V} \propto t^{-\frac{3}{2}(2-\gamma)} \\
& \mathcal{S}_{V} \propto t^{\frac{3}{2} \gamma} .
\end{aligned}
$$

Even though this is derived from the exact solution of type $\mathrm{V}$, one can show that eqs.(10) and (11) will also hold in the generic case (see Appendix A); the expression for Weyl curvature invariant in the type $\mathrm{V}$ case is remarkable simple. Thus, in the type $\mathrm{V}$ case, $P$ is always decreasing and $\mathcal{S}$ is always increasing. Note also that this increase is due to the anisotropic modes; we assumed the universe to be homogeneous.

\footnotetext{
${ }^{6}$ There also exists an $h=4 / 11$ Lukash vacuum solution found by Lukash 36 and a $\gamma=2$ stiff fluid solution by Barrow 37 .
} 
The case $\mathrm{VII}_{h}$ is more difficult. Investigations by Barrow and Sonoda 34, 35, and later by Hewitt and Wainwright [38, 31] indicate that if $2 / 3<\gamma \leq 2$ then the generic solution asymptotes a plane-wave solution at late times. The planewave solutions have $\Sigma=$ constant and zero Weyl curvature invariant. The approach to the plane-wave asymptote, is subtle and a careful investigation of the Weyl curvature invariant in a neighbourhood of the plane-wave solutions is needed. The investigation and calculation is somewhat tedious, but the result is rather interesting:

$$
\left(C^{\alpha \beta \gamma \delta} C_{\alpha \beta \gamma \delta}\right)_{V I I_{h}} \propto t^{-4} \cdot\left[\mathcal{O}\left(t^{-\frac{2\left(1+\Sigma_{+}\right)}{1-2 \Sigma_{+}}}\right)+\mathcal{O}\left(t^{-\frac{4 \Sigma_{+}+(3 \gamma-2)}{1-2 \Sigma_{+}}}\right)\right] .
$$

(see Appendix A on the nature of these asymptotes). Here, $\Sigma_{+}$is a constant parameter which tells us which plane-wave solution is approached as $t \rightarrow \infty$, and $\Sigma_{+}$obeys the inequality

$$
-\frac{1}{4}(3 \gamma-2)<\Sigma_{+}<0
$$

The shear is related to $\Sigma_{+}$for these plane-wave solutions via the simple relation

$$
\Sigma_{\text {plane-wave }}=\left|\Sigma_{+}\right| \text {. }
$$

Note the appearance of the shear-to-volume expansion rate parameter in the power of time rather than as an additive perturbation. This is a signal of the stability of the isotopic solution being decided at second (or higher) order because the Lukash plane-waves are exact solutions of both the Einstein equations and the linearised Einstein equations. Thus a linearisation of the Einstein equations about the Lukash solution gives a zero eigenvalue [35]. Which of the two terms that in eq. (12) is dominant depends on the parameters $\Sigma_{+}$and $\gamma$. The Ricci scalar squared will in general decay as

$$
\left(R^{\mu \nu} R_{\mu \nu}\right)_{V I I_{h}} \propto t^{-4} \cdot t^{-2 \frac{4 \Sigma_{+}+(3 \gamma-2)}{1-2 \Sigma_{+}}} .
$$

Hence,

$$
P_{V I I_{h}}=\mathcal{O}\left(t^{\frac{3\left(\Sigma_{+}+(\gamma-1)\right)}{1-2 \Sigma_{+}}}\right)+\mathcal{O}\left(t^{\frac{4 \Sigma_{+}+(3 \gamma-2)}{2\left(1-2 \Sigma_{+}\right)}}\right)
$$

and the volume expands as $\sqrt{|g|} \propto t^{3 /\left(1-2 \Sigma_{+}\right)}$, so $\mathcal{S}$ will increase as

$$
\mathcal{S}_{V I I_{h}}=\mathcal{O}\left(t^{\frac{3\left(\Sigma_{+}+\gamma\right)}{1-2 \Sigma_{+}}}\right)+\mathcal{O}\left(t^{\frac{4 \Sigma_{+}+3 \gamma}{2\left(1-2 \Sigma_{+}\right)}}\right)
$$

Note that both $P$ and $\mathcal{S} \rightarrow \infty$ as $t \longrightarrow \infty$. Both the Weyl invariant and the Ricci invariants approach zero, but the Weyl invariant does so at a slower rate than the Ricci invariant. Ricci has $R^{\mu \nu} R_{\mu \nu} \propto \rho^{2}$, while the Weyl tensor contains shear modes as well.

The work of Collins and Hawking [39] is of relevance here. This was a study of the stability of isotropic ever-expanding universes against spatially homogeneous perturbations of Bianchi type $\mathrm{VII}_{h}$ and was not restricted to perfect fluids and comoving fluids. In the case of $\mathrm{VII}_{h}$ perturbations to open FRW universes, isotropy was shown to be unstable. However, this well known result requires 
careful interpretation. The definition of stability used was asymptotic stability in the sense of Lyapunov; that is, as $t \rightarrow \infty$ we need to have $\Sigma \rightarrow 0$. However, in general we have found that these $\mathrm{VII}_{h}$ perturbations of the open FRW universe approach the Lukash plane-wave spacetimes which have $\Sigma=$ constant $(\Sigma \rightarrow \infty$ is forbidden by $\rho>0$ ). Thus although isotropy is not asymptotically stable it is stable in the sense that the deviations from isotropy $(\Sigma=0)$ are always bounded [40, 7]. Note that the strong theorem of Collins and Hawking, assuming only the strong energy condition $(\gamma>2 / 3)$ is a reflection of the property that 'the matter doesn't matter' as $t \rightarrow \infty$ in the open universe case. Thus, the behaviour determining the asymptotic stability is that of the vacuum solution and so does not have any significant impact upon why the present anisotropy is so low (see 141 for a discussion of how this may be explained). By contrast, in the case of type $\mathrm{VII}_{0}$ perturbations around the flat FRW model, the matter content of the universe is very influential and $\Sigma$ decays to zero as $t \rightarrow \infty$ only if the dominant form of matter is a perfect fluid with zero pressure $(\gamma=1)$ [39]. Again, this is not very relevant to the present state of the universe since in the absence of inflation the bulk of its expansion e-folds since the Planck epoch have been in a radiation-dominated state for which isotropy is not stable in the Collins and Hawking sense.

The space $\mathbb{H}^{3}$ is infinite, and in the above cases we have assumed that we are dealing with an infinite and unbounded space $\mathbb{H}^{3}$. However, if we relax the assumption of global homogeneity and isotropy, we can get other spacetimes whose spatial section at each instant of time is a compact hyperbolic manifold (CHM). Interestingly, our earlier discussion must be completely revised in the compact case. The compact hyperbolic spaces are heavily constrained by Mostow's rigidity theorem; no anisotropic deformations of CHMs are allowed. All the Bianchi models of type $\mathrm{V}$ and $\mathrm{VII}_{h \neq 0}$ with compact spatial sections have to be (locally) isotropic 24. Hence, their Weyl tensors identically vanish.]

A more general investigation of the behaviour of $\mathcal{S}$ for CHMs is dependent on a calculation of the inhomogeneous eigenmodes of the CHM. This has not been done by date, but some numerical studies have been made 43.

\subsection{The case $S^{3}$}

Turning our attention to the closed universe case, the Bianchi type IX model has the possibility of chaotic dynamics. A closed universe will recollapse to a second singularity after a finite amount of proper time has elapsed if the matter obeys the strong energy condition, the positive pressure criteria, the dominant energy condition, and a matter regularity condition [44, 45]. There can be an asymmetry between behaviour at the initial and final singularities which reflects the relative likelihood of different initial conditions. A special initial state that is chosen close to isotropy will deviate increasingly from isotropy on approach

\footnotetext{
${ }^{7}$ Inhomogeneous deformations are still allowed, but the spherical symmetry of $\mathbb{H}^{3}$ is broken at the global scale when we go to its compact quotients. Thus, the inhomogeneous TolmanBondi model, which we discussed in refs. 10, 42], must also be constrained. The matter content of the Tolman-Bondi models is determined by the dust-density $\rho(r, t)$. If $\rho$ is allowed to vary only as a function of the radius $r$ inside a spherical shell of radius $r_{i n j}$, where $r_{i n j}$ is the coordinate injectivity radius of the CHM, then the compactification can be done. Outside the shell of radius $r_{i n j}$, the density $\rho$ must be constant as a function of $r$ so that the homogeneity can be "restored" outside this shell. The same arguments as for the general Tolman-Bondi model can now be made, and we get a $\mathcal{S} \propto t^{3}$ increase inside $r_{i n j}$.
} 
to any final singularity and exhibit chaotic spacetime oscillations induced by the anisotropy of the three-curvature. However, a quasi-isotropic initial state can be made general by the assumption that the matter content at the initial singularity is dominated by the influence of a $\gamma=2$ fluid or a scalar field with zero potential [37. In this case the difference between the quantity $\mathcal{W}$ and $P$ is also manifest. The quantity $P$ is regular everywhere for the type IX case, except at the singularities. However, at the turning-point of the volume expansion $H=0$, and so $\mathcal{W}$ will diverge there.

When it comes to the initial singularity, the Bianchi type IX has a very peculiar behaviour. Near the initial singularity it displays chaotic behaviour in vacuum and with $\gamma \neq 2$ perfect fluids 446, 47, 48, 49, 50, 51, 52. There is the issue of the coordinate covariance of measures of chaos to be dealt with here, but this problem can be overcome [53]. The geometrical picture is similar to that of the magnetic Bianchi type I universe which we have already discussed. The difference is in the Ricci tensor and in the matter terms. The Weyl curvature invariant will have the same property as in the magnetic Bianchi type I case. If the universe has a perfect fluid with equation of state parameter $\gamma$ then we would expect the same behaviour as in the Kasner universes. Hence,

$$
\mathcal{S}_{I X} \propto t^{\gamma-1}
$$

during the Kasner epochs. The bounces induce downward spikes in $\mathcal{S}$, but on average we will have a steady increase of $\mathcal{S}$ with time until dust domination or potential dominated scalar-field evolution $(\gamma<1$ effectively).

\section{Other models of Thurston type}

\subsection{The case $\widehat{S L(2, \mathbb{R})}$}

Cosmological models with spatial sections $\widehat{S L(2, \mathbb{R})}$ can be invariant under two different Bianchi types, namely III and VIII. If we were to assume a type III invariance with $S L(2, \mathbb{R})$ spatial sections, we would find that this is only compatible if the type III were part of a larger symmetry group. Hence, type III can be considered as a special case of VIII.

Let us therefore investigate models of Bianchi type VIII. When we compactify the spatial space, this model becomes the most generic of the (locally) homogeneous models. The number of free parameters can be arbitrarily large as the topology of the space increases in complexity [24, 25, 26. In addition to this, the type VIII case is interesting for its dynamical behaviour as well. Not only does vacuum and perfect fluid $(\gamma \neq 2)$ type VIII display chaotic behaviour near the initial singularity, as in the IX case, it can also display oscillatory behaviour at late times as in the $\mathrm{VII}_{0}$ evolution [54, 55. So, in this case, two rather peculiar features are manifested together, and by understanding the Bianchi type VIII could possibly be one of the keys for understanding the behaviour of $\mathcal{S}$ and the Weyl tensor in more generic models.

Also the perfect fluid type VIII model has a similar behaviour as the type $\mathrm{VII}_{0}$ [56]. For $1 \leq \gamma<2$ the generic solution will approach the type III form of 
flat space, but it does so in a way such that

$$
\limsup _{t \longrightarrow \infty}\left|\mathcal{W}^{2}\right|=\infty
$$

The shear has the asymptotic value $\Sigma=\frac{1}{4}$ in this case. Thus for the type VIII evolution we have

$$
P_{V I I I} \approx P_{I X}, \quad \mathcal{S}_{V I I I} \approx \mathcal{S}_{I X} \quad \text { as } t \longrightarrow 0
$$

and for $1 \leq \gamma<2$ (actually for $4 / 5<\gamma<2$ )

$$
\overline{\mathcal{W}}_{V I I I}, \bar{P}_{V I I I}, \overline{\mathcal{S}}_{V I I I} \longrightarrow \infty \text { as } t \longrightarrow \infty \text {. }
$$

For $2 / 3<\gamma \leq 4 / 5$, both $\mathcal{W}$ and $\Sigma$ are finite and non-zero at late times; the solutions asymptote to the Collins type $\mathrm{VI}_{-1}$ solution (see section 4.1). We refer to refs [56] and [57] for details.

\subsection{The case Sol}

Solvegeometry has the Bianchi Lie algebra $\mathrm{VI}_{0}$. The solutions will have the Collins $\mathrm{VI}_{0}$ solution as a future attractor when $2 / 3<\gamma<2$. This solution is given by

$$
d s^{2}=-d t^{2}+t^{2} d x^{2}+t^{\frac{2-\gamma}{\gamma}}\left(e^{2 c x} d y^{2}+e^{-2 c x} d z^{2}\right)
$$

where $c \equiv \frac{\sqrt{(2-\gamma)(3 \gamma-2)}}{2 \gamma}$ and $\rho=\frac{(2-\gamma)}{\gamma^{2}} t^{-2}$. The shear is given explicitly by

$$
\Sigma=\frac{(3 \gamma-2)^{2}}{16} \text {. }
$$

We can also calculate the Weyl tensor for this spacetime:

$$
\left(C^{\alpha \beta \gamma \delta} C_{\alpha \beta \gamma \delta}\right)_{V I_{0}}=\frac{2}{3} \frac{(2-\gamma)(3 \gamma-2)^{2}(4-5 \gamma)}{\gamma^{4} t^{4}} .
$$

The Ricci squared scalar is

$$
\left(R^{\mu \nu} R_{\mu \nu}\right)_{V I_{0}}=\frac{(2-\gamma)^{2}\left(3 \gamma^{2}-6 \gamma+4\right)}{\gamma^{4} t^{4}}
$$

and hence,

$$
P_{V I_{0}}^{2}=\frac{(3 \gamma-2)^{2}(4-5 \gamma)}{(2-\gamma)\left(3 \gamma^{2}-6 \gamma+4\right)} .
$$

Unfortunately, $P^{2}<0$ (the magnetic part of the Weyl curvature dominates the electric part) for $\gamma>4 / 5$, which makes $P$ imaginary. Note also that $P=0$ for $\gamma=4 / 5$. Ignoring the sign (taking the absolute value) we can make $P^{2}$ non-negative for all $\gamma$. In this case, we can define $\mathcal{S}$ :

$$
\mathcal{S}_{V I_{0}} \propto t^{\frac{2}{\gamma}} .
$$

Hence, since this is a future attractor solution, the generic solution will behave similarly at late times.

The generic solution starts as a special Kasner solution, and thus $P$ and $\mathcal{S}$ behave as in the Bianchi type I case near the initial singularity.

\footnotetext{
${ }^{8}$ Strictly speaking, these results are not rigorously proven; according to Wainwright they rely on a combination of stability analysis and numerical simulations 56 .
} 


\subsection{The case Nil}

Nil-geometry is invariant under the Bianchi type II group. The future attractor for generic $\gamma$-law perfect fluid solutions with $2 / 3<\gamma<2$ is the Collins-Stewart solution:

$$
d s^{2}=-d t^{2}+t^{\frac{2-\gamma}{\gamma}}\left(d x+\frac{c}{2 \gamma} z d y\right)^{2}+t^{\frac{2+\gamma}{2 \gamma}}\left(d y^{2}+d z^{2}\right)
$$

where $c \equiv \sqrt{(2-\gamma)(3 \gamma-2)}$ and $\rho=(6-\gamma) /\left(4 \gamma^{2} t^{2}\right)$.

The shear and the Weyl curvature invariant can be readily calculated

$$
\begin{aligned}
\Sigma_{I I} & =\frac{(3 \gamma-2)^{2}}{64} \\
\left(C^{\alpha \beta \gamma \delta} C_{\alpha \beta \gamma \delta}\right)_{I I} & \propto t^{-4} .
\end{aligned}
$$

Unfortunately, the Weyl proportionality factor can be negative, positive or zero. Taking the absolute value of the Weyl curvature invariant we obtain

$$
\begin{aligned}
& P_{I I}=\text { constant } \\
& \mathcal{S}_{I I} \propto t^{\frac{2}{\gamma}} .
\end{aligned}
$$

The generic solution near the initial singularity will be approximated by a Kasner solution.

\subsection{The case $\mathbb{H}^{2} \times \mathbb{R}$}

This model corresponds to the Bianchi type III model. In the perfect fluid case, the nature of the future attractor depends on the equation of state parameter $\gamma$. If $\gamma<1$, the future attractor is one of the Collins perfect fluid solutions. If $\gamma \geq 1$ then the generic solutions are future asymptotic to the type III plane-wave solution. Note also that the type III solutions can be considered as a special case of the $\mathrm{VI}_{h}$ solutions for $h=-1$. The $\mathrm{VI}_{h}$ universes will in general be of non-Thurston type and will be considered in section 4.1 .

When $\gamma \geq 1$, the solutions asymptote to a plane-wave solution and so we expect that the Weyl tensor decays faster than $t^{-4}$. Not many exact solutions are known in this case, but the LRS dust and radiation cases are known explicitly. Actually, in the LRS case with $\gamma>1$ we do not need the exact solutions to study the late-time behaviour. We can show that (see Appendix A) if $1<\gamma \leq 3 / 2$, at late times the Weyl tensor will decay as

$$
\left(C^{\alpha \beta \gamma \delta} C_{\alpha \beta \gamma \delta}\right)_{I I I, L R S} \propto t^{-4} \cdot t^{-4(\gamma-1)} .
$$

The late-time asymptotics still have shear and this is given by $\Sigma=1 / 4$. The Ricci tensor will decay similarly, and so

$$
\begin{aligned}
& P_{I I I, L R S}=\text { constant } \\
& \mathcal{S}_{I I I, L R S} \propto t^{2} .
\end{aligned}
$$

The radiation case can be derived explicitly because the exact solutions are known. For $3 / 2 \leq \gamma<2$ the Weyl tensor decays as

$$
\left(C^{\alpha \beta \gamma \delta} C_{\alpha \beta \gamma \delta}\right)_{I I I, L R S} \propto t^{-4} \cdot t^{-2},
$$


$P$ and $\mathcal{S}$ vary as

$$
\begin{aligned}
& P_{I I I, L R S} \propto t^{2 \gamma-3} \\
& \mathcal{S}_{I I I, L R S} \propto t^{2 \gamma-1} .
\end{aligned}
$$

Hence, both $P$ and $\mathcal{S} \rightarrow \infty$ as $t \rightarrow \infty$ for $3 / 2<\gamma \leq 2$.

In the LRS dust case, $\gamma=1$, the Weyl curvature invariant will approach zero at late times as

$$
\left(C^{\alpha \beta \gamma \delta} C_{\alpha \beta \gamma \delta}\right)_{I I I, L R S} \propto t^{-4} \cdot(\ln t)^{-2} .
$$

The Ricci tensor also decreases at the same rate, so at late times $P$ and $\mathcal{S}$ vary as

$$
\begin{aligned}
& P_{I I I, L R S}=\text { constant } \\
& \mathcal{S}_{I I I, L R S} \propto t^{2} \ln t .
\end{aligned}
$$

The non-LRS case is slightly more troublesome. The type III dynamics can be considered as a special case of $\mathrm{VI}_{h}$ with $h=-1$. The generic case is therefore considered in the $\mathrm{VI}_{h}$ section below. The LRS type III case is, as we explain in detail in Appendix A, a special case, and the behaviour of the Weyl curvature invariant is different in the general case than for the LRS type III case. If we want to compactify the type III geometry, we have to take an LRS model. The compactification procedure requires a higher symmetry than the three-dimensional type III symmetry. Hence, compact spatial geometry implies LRS.

In the case $\gamma<1$, the future attractor will be the Collins $\mathrm{VI}_{h}$ perfect fluid solutions with $h=-1$ (see section 4.1). For this solution, the Weyl tensor decays as

$$
\left(C^{\alpha \beta \gamma \delta} C_{\alpha \beta \gamma \delta}\right)_{I I I} \propto t^{-4}
$$

while the shear is constant:

$$
\Sigma_{I I I}=\frac{1}{4}(3 \gamma-2)^{2} .
$$

The Ricci square decays at the same rate, so

$$
\begin{aligned}
& P_{I I I}=\text { constant } \\
& \mathcal{S}_{I I I} \propto t^{\frac{2}{\gamma}} .
\end{aligned}
$$

Near the initial singularity, the solution is approximately that of a Kasner solution. The LRS type III will approach the Taub point $T$ on the Kasner circle. The analysis for the LRS type III shows that near the initial singularity, we get to leading order

$$
\begin{aligned}
\left(C^{\alpha \beta \gamma \delta} C_{\alpha \beta \gamma \delta}\right)_{I I I, L R S} & \propto t^{-4} \cdot t^{2(2-\gamma)} \\
P_{I I I, L R S} & =\text { constant } \\
\mathcal{S}_{I I I, L R S} & \propto t .
\end{aligned}
$$

Hence, the type III behaves like the Bianchi type I universe near the initial singularity. 


\subsection{The case $S^{2} \times \mathbb{R}$}

This includes the closed Kantowski-Sachs case and there is no corresponding Bianchi model. However, all the Kantowski-Sachs solutions are asymptotic to a plane symmetric Kasner model (not $T$ ) as $t \rightarrow 0$. Hence, near the initial singularity, we have

$$
\left(C^{\alpha \beta \gamma \delta} C_{\alpha \beta \gamma \delta}\right)_{K S} \propto t^{-4}
$$

The Ricci square is determined by the square of the energy density, and hence, as $t \rightarrow 0$

$$
\begin{aligned}
& P_{K S} \propto t^{\gamma-2} \\
& \mathcal{S}_{K S} \propto t^{\gamma-1} .
\end{aligned}
$$

The Kantowski-Sachs models will recollapse for many types of fluids 44, 45]. Recent observations do suggest however, that we live in a state of accelerated expansion. This type of behaviour can be explained by the late-time influence of a quintessence scalar field. In these cases, the KS universe can be ever-expanding. The KS models with a cosmological constant $(\gamma=0)$ contain an unusual unstable particular solution which does not approach the de Sitter solution as $t \rightarrow \infty$, [58. The cosmic no hair theorem [59] is not violated because the 3 -curvature is positive. This unusual behaviour has been used to argue for forms of dimensionally selective inflation [60], however, the behaviour is an artefact of the $S^{2} \times \mathbb{R}$ symmetry and is unstable even within the KS class of solutions 61.

\section{Models of non-Thurston type}

\subsection{The Bianchi type $\mathrm{VI}_{h}$.}

For this model, there exist future-attractor solutions for all $\gamma$ (as in the $\mathrm{VI}_{0}$ case). The nature of these attractors depends on the group parameter $h$ (which is non-positive) and on the equation of state parameter $\gamma$. If $\gamma$ satisfies

$$
-h<\frac{2-\gamma}{3 \gamma-2}
$$

then the future attractor solution is the Collins $\mathrm{VI}_{h}$ perfect fluid solution with metric

$$
d s^{2}=-d t^{2}+t^{2} d x^{2}+t^{\frac{2-\gamma}{\gamma}} e^{r x \frac{2-\gamma}{\gamma}}\left(t^{\frac{r c}{\gamma}} e^{2 c x} d y^{2}+t^{-\frac{r c}{\gamma}} e^{-2 c x} d z^{2}\right),
$$

where $c \equiv \sqrt{(2-\gamma)(3 \gamma-2)}$ and $0<r<1$ is related to $h$ by $h=-(2-\gamma)^{2} r^{2} / c^{2}$. These solutions are universes with shear and some of their physical properties have been studied by one of us in connection with primordial nucleosynthesis [62. The shear is found to be

$$
\Sigma_{V I_{h}}=\frac{1}{16}(1-3 h)(3 \gamma-2)^{2} .
$$

The Weyl tensor in this case has a similar behaviour as in the type $\mathrm{VI}_{0}$ case. For some values of $\gamma$ it is negative, and others positive. Ignoring the sign, as we 
did in the $\mathrm{VI}_{0}$ case, we get

$$
\begin{aligned}
\left(C^{\alpha \beta \gamma \delta} C_{\alpha \beta \gamma \delta}\right)_{V I_{h}} & \propto t^{-4} \\
P_{V I_{h}} & =\text { constant } \\
\mathcal{S}_{V I_{h}} & \propto t^{\frac{2}{\gamma}}
\end{aligned}
$$

If the equation of state parameter $\gamma$ fails to obey eq. (21), and so

$$
-h \geq \frac{2-\gamma}{3 \gamma-2}
$$

then the solutions appear to be asymptotic to plane-wave spacetimes? The plane-wave solutions have zero Weyl-curvature invariants, therefore we need to know how these solutions approach the future attractor. Unfortunately, only a few exact $\mathrm{VI}_{h}$ solutions with this behaviour are known.

Nevertheless, we can show that when $\gamma$ obeys the inequality (23), the behaviour of a generic solution is

$$
\left(C^{\alpha \beta \gamma \delta} C_{\alpha \beta \gamma \delta}\right)_{V I_{h}} \propto t^{-4} \cdot\left[\mathcal{O}\left(t^{-\frac{2\left(1+\Sigma_{+}\right)}{1-2 \Sigma_{+}}}\right)+\mathcal{O}\left(t^{-\frac{4 \Sigma_{+}+(3 \gamma-2)}{1-2 \Sigma_{+}}}\right)\right]
$$

where $\Sigma_{+}$characterises the different plane-wave solutions (see Appendix A). The shear is directly related to this parameter via $\Sigma=\left|\Sigma_{+}\right|$. The Ricci square goes as $\rho^{2}$, and hence

$$
P_{V I_{h}}=\mathcal{O}\left(t^{\frac{3\left(\Sigma_{+}+(\gamma-1)\right)}{1-2 \Sigma_{+}}}\right)+\mathcal{O}\left(t^{\frac{4 \Sigma_{+}+(3 \gamma-2)}{2\left(1-2 \Sigma_{+}\right)}}\right)
$$

Interestingly, in general $P$ will grow unbounded as we approach the future attractor. Thus, in these solutions, the Ricci tensor will decrease faster than the shear modes captured by the Weyl curvature invariant. The Weyl tensor will decrease towards a plane-wave state, but as it does so, the Ricci tensor will be sub-dominant compared to the Weyl tensor.

The volume expands as $\sqrt{|g|} \propto t^{3 /\left(1-2 \Sigma_{+}\right)}$, thus $\mathcal{S}$ will increase as

$$
\mathcal{S}_{V I_{h}}=\mathcal{O}\left(t^{\frac{3\left(\Sigma_{+}+\gamma\right)}{1-2 \Sigma_{+}}}\right)+\mathcal{O}\left(t^{\frac{4 \Sigma_{+}+3 \gamma}{2\left(1-2 \Sigma_{+}\right)}}\right)
$$

As mentioned earlier, these solutions are future attractor solutions, and hence, the generic solutions will have a similar behaviour for their Weyl tensors. Near the initial singularity, the generic behaviour is Kasner-like and the Weyl tensor behaves as in the Bianchi type I.

\subsection{The exceptional case}

The exceptional case of Bianchi type $\mathrm{VI}_{-1 / 9}^{*}$, has to be treated separately. The exceptional case has as many free parameters as the whole family $\mathrm{VI}_{h}$ with arbitrary $h$ because the choice $h=-1 / 9$ results in the disappearance of some of the Einstein constraint equations. Unfortunately, the exceptional case has not

\footnotetext{
${ }^{9}$ This is not rigorously proven, but Hewitt and Wainwright 38,31 have conjectured that, except for sets of measure zero, these plane-wave solutions are global future attractors. The same is also the case for types IV and $\mathrm{VII}_{h}$.
} 
been much studied in the literature. Stability properties were studied by Barrow and Sonoda [35], a study of the consequences for primordial nucleosynthesis was made in [62], and Hewitt [63], studied the evolution in a subspace of the whole dynamical system of $\mathrm{VI}_{-1 / 9}^{*}$ (namely the subspace with $n_{\alpha}^{\alpha}=0$ ). In this subspace Hewitt and Barrow and Sonoda showed that for $2 / 3<\gamma<10 / 9$, $\gamma=10 / 9$ and $10 / 9<\gamma<2$ the future attractors were the Collins $\mathrm{VI}_{-1 / 9}$ perfect fluid solutions, Wainwright $\gamma=10 / 9$ solutions [64] with metric

$$
d s^{2}=-d t^{2}+t^{2} d x^{2}+t^{\frac{2}{5}}\left(e^{A x} d y+w t^{\frac{4}{5}} d x\right)^{2}+t^{\frac{6}{5}} e^{-4 A x} d z^{2}
$$

where $w^{2}=9 r^{2} / 4-1$ and $A=r \sqrt{6} / 5$, and the Robinson-Trautman vacuum solution $[0$ with metric [35]

$$
d s^{2}=-d t^{2}+\frac{75}{8} t^{2} d x^{2}+\frac{5 \sqrt{10}}{8} e^{-x} t^{\frac{6}{5}} d x d y+\frac{3}{16} e^{-2 x} t^{\frac{2}{5}} d y^{2}+e^{4 x} t^{\frac{6}{5}} d z^{2},
$$

respectively. We can readily calculate the Weyl tensors for these spacetimes. For $2 / 3<\gamma \leq 10 / 9$ we get

$$
\left(C^{\alpha \beta \gamma \delta} C_{\alpha \beta \gamma \delta}\right)_{V I_{-1 / 9}^{*}} \propto t^{-4} .
$$

The Ricci tensor decays at the same rate, and hence

$$
\begin{aligned}
& P_{V I_{-1 / 9}^{*}} \propto \text { constant } \\
& \mathcal{S}_{V I_{-1 / 9}^{*}} \propto t^{\frac{2}{\gamma}} .
\end{aligned}
$$

The Robinson-Trautman solution corresponds to a universe with shear $\Sigma=$ $1 / 3$, but has a zero Weyl curvature scalar. However, when $10 / 9<\gamma<2$ we can show that for any generic solution that approaches this solution, we have

$$
\left(C^{\alpha \beta \gamma \delta} C_{\alpha \beta \gamma \delta}\right)_{V I_{-1 / 9}^{*}} \propto t^{-4} \cdot\left[\mathcal{O}\left(t^{-\frac{2}{3}}\right)+\mathcal{O}\left(t^{-\frac{9 \gamma-10}{3}}\right)\right]
$$

(see Appendix B). Note that the two terms are equally dominant when $\gamma=4 / 3$ (radiation). At late times the Ricci square decays faster towards zero than the Weyl curvature invariant:

$$
\begin{aligned}
& P_{V I_{-1 / 9}^{*}} \propto \mathcal{O}\left(t^{\frac{9 \gamma-11}{3}}\right)+\mathcal{O}\left(t^{\frac{9 \gamma-10}{6}}\right) \\
& \mathcal{S}_{V I_{-1 / 9}^{*}} \propto \mathcal{O}\left(t^{3\left(\gamma-\frac{28}{45}\right)}\right)+\mathcal{O}\left(t^{\frac{3}{2}\left(\gamma+\frac{4}{45}\right)}\right) .
\end{aligned}
$$

In this case the Weyl curvature invariant will dominate at late times. At early times, the generic solution approaches a certain point on the Kasner circle (not $T$ ), and hence, the analysis of Bianchi type I applies again.

As mentioned, this analysis is just for a special subspace in the dynamical state space of $\mathrm{VI}_{-1 / 9}^{*}$. Nobody, at least to the authors' knowledge, has made a complete analysis of the whole dynamical behaviour of the exceptional case. However, page 171 in [31] appears to indicate that the exceptional case has a chaotic initial singularity. The future attractor seems to be a self-similar spacetime, and hence the above results may hold in that case. Further investigation of this case is required.

\footnotetext{
${ }^{10}$ This solution is also sometimes called the Collinson-French solution. It contains no free parameters.
} 


\subsection{The Bianchi type IV}

It is conjectured that generically the Bianchi type IV solutions move asymptotically towards plane-wave solutions. The behaviour is similar to the $\mathrm{VI}_{h}$ case, and the late-time behaviour for the Weyl tensor is similar to the plane-wave case. Hence, we will have

$$
\left(C^{\alpha \beta \gamma \delta} C_{\alpha \beta \gamma \delta}\right)_{I V} \propto t^{-4} \cdot\left[\mathcal{O}\left(t^{-\frac{2\left(1+\Sigma_{+}\right)}{1-2 \Sigma_{+}}}\right)+\mathcal{O}\left(t^{-\frac{4 \Sigma_{+}+(3 \gamma-2)}{1-2 \Sigma_{+}}}\right)\right]
$$

(see Appendix A). Again the shear is given by $\Sigma=\left|\Sigma_{+}\right|$. The Ricci square goes as $\rho^{2}$, and hence

$$
P_{V I_{h}}=\mathcal{O}\left(t^{\frac{3\left(\Sigma_{+}+(\gamma-1)\right)}{1-2 \Sigma_{+}}}\right)+\mathcal{O}\left(t^{\frac{4 \Sigma_{+}+(3 \gamma-2)}{2\left(1-2 \Sigma_{+}\right)}}\right)
$$

Finally, we have

$$
\mathcal{S}_{V I_{h}}=\mathcal{O}\left(t^{\frac{3\left(\Sigma_{+}+\gamma\right)}{1-2 \Sigma_{+}}}\right)+\mathcal{O}\left(t^{\frac{4 \Sigma_{+}+3 \gamma}{2\left(1-2 \Sigma_{+}\right)}}\right)
$$

So, in this case the $P$ and $\mathcal{S} \rightarrow \infty$ at late times. Near the initial singularity, the type IV behaves like a Kasner universe, and hence, the Bianchi type I analysis applies again.

\section{Conclusion}

In this paper we have investigated the general behaviour of the Weyl curvature invariant for spatially homogeneous universes containing a perfect fluid as $t \rightarrow 0$ and $t \rightarrow \infty$. For all the models, except for the chaotic ones, the solutions asymptote in the past to points on the Kasner circle. The Kasner universe, which is a special vacuum case of the Bianchi type I model, has been treated exhaustively elsewhere [27, 10].

In the chaotic models, we saw that the Weyl curvature invariant $C^{\alpha \beta \gamma \delta} C_{\alpha \beta \gamma \delta}$, the invariant $P^{2}=\frac{C^{\alpha \beta \gamma \delta} C_{\alpha \beta \gamma \delta}}{R^{\mu \nu} R_{\mu \nu}}$, and the invariant $\mathcal{S}=\sqrt{|g|} P$ experience peaks and sinks. On the average, however, $\mathcal{S}$ increases steadily as the universe expands from the initial singularity.

The late-time behaviour was found to fall into one of five categories:

1. Ricci dominance. This category includes models for which $P$ is decreasing at late times, indicating that the Ricci square dominates over the Weyl curvature invariant asymptotically.

2. Weyl-Ricci balanced. In this category $P$ is approximately constant at late times. The Ricci and Weyl tensors are approximately proportional at late times.

3. Weyl dominance. In this category $P \rightarrow \infty$ as $t \rightarrow \infty$, but the expansionnormalized Weyl tensor, $\mathcal{W}^{2}=C^{\alpha \beta \gamma \delta} C_{\alpha \beta \gamma \delta} / H^{4}$, will decrease. Except in the exceptional $\mathrm{VI}_{-1 / 9}^{*}$ case, these models evolve towards vacuum planewave spacetimes, but the Weyl curvature invariant decreases more slowly than the Ricci square. 


\begin{tabular}{|c|c|c|}
\hline Type & Matter & Category \\
\hline \hline I & $2 / 3<\gamma \leq 2$ & 1 \\
& $2 / 3<\gamma \leq 4 / 3+\pi_{\mu \nu}$ & 1 \\
& $4 / 3<\gamma \leq 2+$ mag.field & 2 \\
\hline II & $2 / 3<\gamma<2$ & 2 \\
\hline III $_{L R S}$ & $2 / 3<\gamma \leq 3 / 2$ & 2 \\
& $3 / 2<\gamma \leq 2$ & 3 \\
\hline IV & $2 / 3<\gamma \leq 2$ & 3 \\
\hline V & $2 / 3<\gamma \leq 2$ & 1 \\
\hline $\mathrm{VI}_{0}$ & $2 / 3<\gamma<2$ & 2 \\
\hline $\mathrm{VI}_{h}$ & $2 / 3<\gamma<\frac{2(1-h)}{1-3 h}$ & 2 \\
& $\frac{2(1-h)}{1-3 h} \leq \gamma \leq 2$ & 3 \\
\hline $\mathrm{VI}_{-1 / 9}^{*}$ & $2 / 3<\gamma \leq 10 / 9$ & 2 \\
& $10 / 9<\gamma \leq 2$ & 3 \\
\hline $\mathrm{VII}_{0}$ & $2 / 3<\gamma<1$ & 1 \\
& $1<\gamma \leq 2$ & 4 \\
\hline $\mathrm{VII} h$ & $2 / 3<\gamma \leq 2$ & 3 \\
\hline VIII & $2 / 3<\gamma<4 / 5$ & 2 \\
& $4 / 5<\gamma \leq 2$ & 4 \\
\hline IX & $2 / 3<\gamma \leq 2$ & 5 \\
\hline $\mathrm{KS}$ & $2 / 3<\gamma \leq 2$ & 5 \\
\hline $\mathrm{I}-\mathrm{IX}$ & $0 \leq \gamma<2 / 3$ & 1 \\
\hline
\end{tabular}

Table 1: Classification of the late-time behaviour. The matter content of the universe is labelled by the perfect fluid equation of state parameter, $\gamma ; \pi_{\mu \nu}$ indicates the presence of trace-free anisotropic stresses and type I also includes the case of a pure magnetic field.

4. Extreme Weyl dominance. In these models, $\mathcal{W} \rightarrow \infty$ as $t \rightarrow \infty$.

5. Recollapsing models. These models have no late-time behaviour, they recollapse after a finite time subject to the usual technical energy conditions required for recollapse [44].

In the Table 11 we have summarised our results, and in the rightmost column we have indicated to which of the five categories each of the spatially homogeneous models belong. We note that category-3 cosmologies are not often discussed in the literaturem. All of the plane-wave solutions of class B belong to this category. Note also that when we compactify the topology of the spatial sections [24, 25], most of the models in this category disappear in the compactification procedure; types IV and $\mathrm{VI}_{h}$ cannot be compactified, type III must be locally rotationally symmetric (LRS), and $\mathrm{VII}_{h}$ must be isotropic. However, some of the type III spacetimes (namely those LRS with $\gamma>3 / 2$ ) in category 3 can be compactified.

A general criterion to decide when a dynamical system will end up in category 3 can be given. If the late-time asymptote is a vacuum spacetime which is not conformally flat, but nevertheless has a zero Weyl curvature invariant, then

\footnotetext{
${ }^{11}$ This behaviour seems to have been noted for a special class of the $\mathrm{VI}_{h}$ solutions in [18].
} 
the quantity $P$ will generically increase without bound. If, in addition, the state space of the corresponding dynamical system is also compact, then $\mathcal{W}$ has to be bounded (and thus decreasing), and hence, the dynamical system will end up in category 3 . The reason for this is as follows. For a conformally flat spacetime, both the Weyl curvature invariant and the Weyl tensor itself vanish. Hence, any linear perturbation of this conformally flat solution will produce linear corrections to the components of the Weyl tensor. Since the linear terms are the lowest-order terms in the Weyl tensor, quadratic terms will be the lowest-order terms in the Weyl curvature invariant. For non-conformally flat spacetimes, the Weyl curvature tensor will not be zero. Hence, the linear terms will not be the lowest-order terms in the perturbation. Thus, in the Weyl curvature invariant some of the linear terms will survive even though the lowest-order (constant) terms could vanish identically (which happens for the plane-wave solutions). The electric and the magnetic parts of the Weyl tensor are not zero for planewave solutions, they just happen to be of equal magnitude and cancel when the invariant is formed.

If the late-time asymptote is conformally flat, but not Ricci flat (hence, nonvacuum), then the system will typically end up in category 1 . There is also a very small chance that the spacetime will end up in category 4 if the state space is non-compact. If the asymptote is both Ricci flat and conformally flat, all five categories are possible. If the state space is compact, then category 4 is ruled out. The fifth category depends to a certain extent on the geometry of the late-time attractor. For instance, inflationary spacetimes with $\rho+3 p<0$, are all governed by the "no hair" theorem which states that the late-time asymptote is a conformally flat non-vacuum spacetime. Hence, all ever-inflating cosmologies should end up in category 1.

In all of the models investigated, except for those in category $5, \mathcal{S}$ increases at late times. Hence, $\mathcal{S}$ behaves as expected by the WCC. It is not clear whether we should always expect a Weyl 'entropy' to increase if the initial conditions are close to some state of effective gravitational equilibrium. Of course, our study has only discussed spatially homogeneous universes and it remains to be seen whether small density and gravitational wave inhomogeneities evolve in a manner consistent with a thermodynamic interpretation of the Weyl curvature and whether the inclusion of significant inhomogeneities, black holes, and damping processes introduces significant new ingredients to the search for the elusive gravitational 'entropy'.

\section{Appendix A: The Weyl-Curvature Invariant for Bianchi types of class B.}

We will briefly show how the Weyl curvature invariant behaves near the planewave equilibrium points of type III, $\mathrm{V}, \mathrm{VI}_{h}, \mathrm{IV}$ and $\mathrm{VII}_{h}$. The discussion is based on Hewitt and Wainwright's approach [31, 38] (see also [35] and 65, 66]).

For all the plane-wave solutions we will have $C_{\alpha \beta \gamma \delta} C^{\alpha \beta \gamma \delta}=0$, so the question we would like to answer is how the Weyl curvature invariant approaches zero at late times. The Weyl curvature invariant is given explicitly by

$$
C_{\alpha \beta \gamma \delta} C^{\alpha \beta \gamma \delta}=8 H^{4}\left[\left(\mathcal{E}_{A B} \mathcal{E}^{A B}+6 \mathcal{E}_{+}^{2}\right)-\left(\mathcal{H}_{A B} \mathcal{H}^{A B}+6 \mathcal{H}_{+}^{2}\right)\right]
$$


where the expansion-normalised electric parts and magnetic parts of the Weyl curvature are given by

$$
\begin{aligned}
\mathcal{E}_{+}= & \Sigma_{+}\left(1+\Sigma_{+}\right)-\tilde{\Sigma}+\frac{2}{3} N_{+}^{2}-\frac{2}{3} \tilde{h} \tilde{A} \\
\mathcal{H}_{+}= & 3 \Delta \\
\mathcal{E}_{A B} \mathcal{E}^{A B}= & 6\left[\tilde{\Sigma}\left(2 \Sigma_{+}-1\right)^{2}+4\left\{\tilde{A} \Sigma_{+}+\frac{1}{3}\left(N_{+}^{2}-\tilde{h} \tilde{A}\right)\left(\tilde{A}+\left(N_{+}^{2}-\tilde{h} \tilde{A}\right)-2 \tilde{\Sigma}\right)\right\}\right. \\
& \left.+4 \Delta^{2}-4 N_{+} \Delta\left(2 \Sigma_{+}-1\right)+\frac{4}{3} \tilde{h} \tilde{A}\left(N_{+}^{2}-\tilde{h} \tilde{A}\right)\right] \\
\mathcal{H}_{A B} \mathcal{H}^{A B}= & 6\left[3 \Sigma_{+}^{2}\left(N_{+}^{2}-\tilde{h} \tilde{A}\right)+2 \tilde{\Sigma}\left(N_{+}^{2}-\tilde{h} \tilde{A}\right)+\tilde{\Sigma} \tilde{A}+6 \Delta^{2}\right. \\
& \left.+12 \Sigma_{+} N_{+} \Delta+4 \tilde{h} \tilde{A} \tilde{\Sigma}\right] .
\end{aligned}
$$

The shear is given by

$$
\Sigma=\widetilde{\Sigma}+\Sigma_{+}^{2} .
$$

All the variables and their interpretations are explained in detail in refs. [31, 38]. In particular, $\tilde{h}$ is related to the Lie algebra parameter $h$ via $\tilde{h}=1 / h$. The Bianchi types are related to $\tilde{h}$ as follows.

$$
\begin{array}{ll}
\tilde{h}<0: & \mathrm{VI}_{h} \\
\tilde{h}=0: & \mathrm{IV} \\
\tilde{h}>0: & \mathrm{VII}_{h} .
\end{array}
$$

Note that type III is $\mathrm{VI}_{-1}$ and type $\mathrm{V}$ is contained in the discussion as the special case of $\tilde{h}=0$.

In these variables the plane-wave equilibrium points are given by

$$
\begin{aligned}
\tilde{\Sigma} & =-\Sigma_{+}\left(1+\Sigma_{+}\right), \quad \Delta=0, \quad \tilde{A}=\left(1+\Sigma_{+}\right)^{2}, \\
N_{+}^{2} & =\left(1+\Sigma_{+}\right)\left[\tilde{h}\left(1+\Sigma_{+}\right)-3 \Sigma_{+}\right],
\end{aligned}
$$

where $\Sigma_{+}$is a parameter which satisfies

$$
\begin{gathered}
-1<\Sigma_{+} \leq \frac{\tilde{h}}{3-h}, \quad \text { if } \tilde{h}<0 \\
-1<\Sigma_{+}<0, \quad \text { if } \tilde{h} \geq 0
\end{gathered}
$$

The eigenvalues of the dynamical system for these equilibrium points are given by

$$
\left\{0, \quad-4 \Sigma_{+}-(3 \gamma-2), \quad-2\left[\left(1+\Sigma_{+}\right) \pm i N_{+}\right]\right\},
$$

where $N_{+}$is given by (29). The equilibrium points are future attractors whenever

$$
\Sigma_{+}>-\frac{1}{4}(3 \gamma-2) .
$$

The second of these eigenvalues is directly related to the time evolution of the perfect fluid. If $\Omega$ is the expansion-normalised energy density, $\Omega$ will in a close neighbourhood of the plane-wave equilibrium points obey

$$
\Omega^{\prime}=\left[-4 \Sigma_{+}-(3 \gamma-2)\right] \Omega
$$




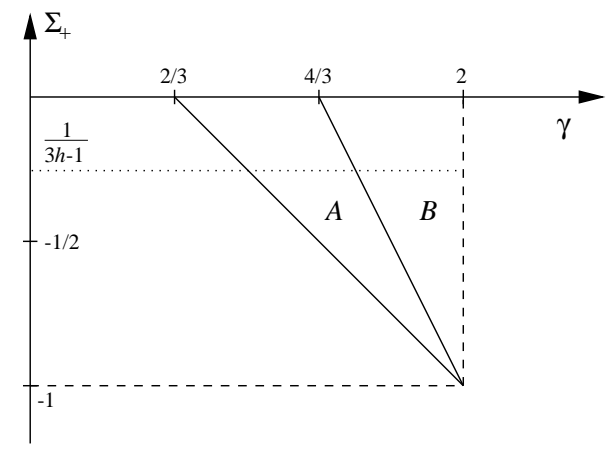

Figure 1: In region $A$ the eigenvalue $-4 \Sigma_{+}-(3 \gamma-2)$ is larger than $-2\left(\Sigma_{+}+1\right)$. In region $B,-2\left(\Sigma_{+}+1\right)$ is the largest.

where prime denotes derivation with respect to the time variable $\tau$ defined by

$$
\frac{d t}{d \tau}=\frac{1}{H}
$$

For the plane-wave solutions, we have $\Omega=0$. The dependence of the eigenvalues on the equation of state parameter is shown in Figure 1.

Upon a small variation of the variables from these equilibrium points, the variation will decay as linear combination of factors $\exp \left(\lambda_{i} \tau\right)$ where $\lambda_{i}$ are the eigenvalues (31). Hence, we would naively expect the Weyl curvature to behave as

$$
\mathcal{W}^{2}=\mathcal{O}\left(\exp \left(\lambda_{i} \tau\right)\right)
$$

But here we have to be careful. It can be shown that the generic behaviour of the Weyl curvature invariant is like this, but in some important cases it is not. For instance, for the radiative solution of LRS type III which we mentioned in section 3.4, all the linear terms from the variation around the plane-wave solution vanish. If we constrain ourself to lie in the invariant subspace $\Delta=$ $\Sigma_{+} N_{+}, 3 \Sigma_{+}^{2}+\tilde{h} \tilde{\Sigma}=0, \tilde{A}>0$, then we would get

$$
\begin{aligned}
\mathcal{W}^{2}= & \frac{9}{(3-\tilde{h})}\left[(1+\tilde{h})\left\{(12 \tilde{h}+7) \Omega+(69 \tilde{h}-121) \sigma_{+}\right\}+\sqrt{1+\tilde{h}}(27 \tilde{h}+5) \nu\right. \\
& \left.\left.+ \text { (quadratic terms in } \Omega, \sigma_{+}, \nu\right)\right] .
\end{aligned}
$$

Here, $\Omega$ is a small variation of the density of the perfect fluid, and $\sigma_{+}$and $\nu$ are small variations of $\Sigma_{+}$and $N_{+}$respectively. The LRS type III is precisely this invariant subspace when $\tilde{h}=-1$. Note that if and only if $h=\tilde{h}=-1$, the linear terms in $\Omega, \sigma_{+}$and $\nu$ will vanish identically. In the case were $\gamma<3 / 2, \Omega$ will dominate at late times over $\sigma_{+}$and $\nu$, thus

$$
\mathcal{W}^{2} \propto \Omega^{2}
$$

at late times. Note also that $R^{\mu \nu} R_{\mu \nu} \propto H^{4} \cdot \Omega^{2}$ which means that $P_{I I I} \approx$ constant at late times for these LRS type III solutions. 
The type $\mathrm{V}$ model is of special interest. The invariant subspace is given by $\tilde{h}=0, \tilde{A}>0$ while $\Sigma_{+}=\Delta=N_{+}=0$. In this case the Weyl curvature invariant is particularly simple:

$$
\mathcal{W}_{V}^{2}=48\left(2 \tilde{\Sigma}^{2}+\tilde{\Sigma} \Omega\right) .
$$

For completeness, we should mention that for all of the plane-wave solutions we have a simple inversion time evolution for the mean Hubble parameter. The simple Milne evolution arises only when the shear parameter $\Sigma_{+}=0$ :

$$
\begin{aligned}
t & \propto e^{\left(1-2 \Sigma_{+}\right) \tau} \\
H & =\frac{1}{1-2 \Sigma_{+}} t^{-1} .
\end{aligned}
$$

\section{Appendix B: The Weyl-Curvature Invariant in the exceptional case of Bianchi type $\mathrm{VI}_{-\frac{1}{9}}^{*}$}

For the dynamical system of Hewitt [63], the Weyl tensor is

$$
\begin{aligned}
\mathcal{W}^{2}= & \frac{8}{3} \Sigma_{+}^{2}\left(3+18 \Sigma_{12}^{2}+4 \Sigma_{+}^{2}-16 N_{\times}^{2}\right) \\
& +32 N^{2}\left(\Sigma_{+}+N_{\times}^{2}-3 \Sigma_{12}^{2}\right)+18 \Sigma_{12}^{2}\left(1+3 \Sigma_{12}^{2}\right)
\end{aligned}
$$

while the shear is

$$
\Sigma=\frac{4}{3} \Sigma_{+}^{2}+3 \Sigma_{12}^{2}
$$

The Robinson - Trautman vacuum solution has

$$
\Sigma_{+}=-\frac{1}{3}, \quad \Sigma_{12}=\frac{\sqrt{5}}{9}, \quad N_{\times}=\frac{\sqrt{2}}{2} .
$$

The eigenvalues of the linear system with respect to this equilibrium point are given by [63], [35]

$$
-\frac{2}{3}(1 \pm \sqrt{14} i), \quad \frac{10-9 \gamma}{3} .
$$

In this case, the Weyl tensor will have linear terms in all of the three variables. Thus the Weyl tensor will decay as $\exp \left(\lambda_{\max } \tau\right)$ where $\lambda_{\max }$ is the eigenvalue with the largest real part. The time variable $\tau$ is related with the cosmological time via

$$
t \propto e^{\frac{5}{3} \tau}
$$

near the equilibrium point.

\section{Acknowledgments}

We deeply appreciate the useful and insightful comments made by $\varnothing$. Grøn, S. Siklos and Y. Gaspar. SH was funded by the Research Council of Norway. 


\section{References}

[1] J.D. Bekenstein, Phys. Rev. D7 (1973) 2333.

[2] S.W. Hawking, Comm. Math. Phys., 43 (1975) 199.

[3] P. Candelas and D.W. Sciama, Phys. Rev. Lett. 38 (1977) 1372

[4] B.L. Hu, Phys. Lett. 97A (1983) 368

[5] P.C.W. Davies, The Physics of Time Asymmetry, Univ. of California Press, Berkeley (1974).

[6] P.C.W. Davies, Nature, 301 (1983) 398.

[7] J.D. Barrow and F.J. Tipler, The Anthropic Cosmological Principle, Oxford University Press (1986)

[8] R. Penrose, in General Relativity, an Einstein centenary survey, eds. S.W. Hawking and W. Israel, Cambridge Univ. Press (1979).

[9] J. Wainwright and P.J. Anderson, Gen. Rel. Grav. 16 (1984) 609.

[10] Ø. Grøn and S. Hervik, Class. Quantum Grav. 18 (2001) 601.

[11] Ø. Grøn and S. Hervik, gr-qc/0205026 to appear in Int. J. Theo. Phys. Group Th. Nonlin. Opt.

[12] T. Rothman and P. Anninos, Phys. Lett. A224 (1997) 227.

[13] T. Rothman, Gen. Rel. Grav. 32 (2000) 1185.

[14] S.W. Goode and J. Wainwright, Class. Quantum Grav. 2 (1984) 99.

[15] W.B. Bonnor, Phys. Lett. 112A (1985) 26.

[16] V. Husain, Phys. Rev. D38 (1988) 3314.

[17] N. Pelavas and K. Lake, Phys. Rev. D62 (2000) 044009.

[18] S.W. Goode, A.A. Coley and J. Wainwright, Class. Quantum Grav. 9 (1992) 445

[19] W.P. Thurston, Three-Dimensional Geometry and Topology, Princeton Uni. Press (1997)

[20] W.P. Thurston, Bull. Am. Math. Soc. 6 (1982) 357

[21] A. Ashtekar and J. Samuel, Class. Quantum Grav. 8 (1991) 2191

[22] Y. Fujiwara, H. Ishihara and H. Kodama, Class. Quantum Grav. 10 (1993) 859

[23] H. Kodama, Prog. Theor. Phys. 99 (1998) 173

[24] J.D. Barrow and H. Kodama, Class. Quantum Grav. 18 (2001) 1753

[25] J.D. Barrow and H. Kodama, Int. J. Mod. Phys. D 10 (2001) 785 
[26] H. Kodama, gr-qc/0109064

[27] S. Hervik, Class. Quantum Grav. 17 (2000) 2765.

[28] S. Hervik, Gen. Rel. Grav. 33 (2001) 2027

[29] V.G. LeBlanc, Class. Quantum Grav. 14 (1997) 2281

[30] J.D. Barrow and J. Levin, Phys. Rev. Lett. 80 (1998) 656.

[31] C.G. Hewitt and J. Wainwright in Dynamical Systems in Cosmology, eds: J. Wainwright and G.F.R. Ellis, Cambridge University Press (1997)

[32] J. Wainwright, M.J. Hancock and C. Uggla, Class. Quantum Grav. 16 (1999) 2577

[33] U.S. Nilsson, M.J. Hancock and J. Wainwright, gr-qc/9912019

[34] J.D. Barrow and D.H. Sonoda, Gen. Rel. Grav. 17 (1985) 409

[35] J.D. Barrow and D.H. Sonoda, Phys. Reports 139 (1986) 1

[36] V. Lukash, Sov. Phys. JETP 40 (1975) 792

[37] J.D. Barrow, Nature 272 (1978), 211

[38] C.G. Hewitt and J. Wainwright, Class. Quantum Grav., 10 (1993) 99

[39] C.B. Collins and S.W. Hawking, Astrophys. J. 180 (1973) 317

[40] J.D. Barrow, Quart. Jl. Roy. astr. Soc. 23 (1982) 344

[41] J.D. Barrow, Phys. Rev. D51 (1995) 3113

[42] S. Hervik, Class. Quantum Grav. 18 (2001) 175

[43] K.T. Inoue, Class. Quantum Grav. 18 (2001) 629

[44] J.D. Barrow, G.J. Galloway and F.J. Tipler MNRAS 223 (1986) 835

[45] J.D. Barrow and F.J. Tipler MNRAS 216 (1985) 395

[46] V. A. Belinskii, I. M. Khalatnikov and E. M. Lifshitz, Adv. Phys. 19 (1970) 525

[47] J.D. Barrow, Phys. Rev. Lett. 46 (1981) 963

[48] J.D. Barrow, Phys. Reports 85 (1982) 1

[49] D. Chernoff and J.D. Barrow, Phys. Rev. Lett. 50 (1983) 134

[50] J. Demaret, M. Henneaux, and P. Spindel, Phys. Lett. B 164 (1985) 27

[51] T. Damour, M. Henneaux, B. Julia and H. Nicolai, Phys. Lett. B 509 (2001) 323

[52] T. Damour and M. Henneaux, Phys. Rev. Lett. 86 (2001) 4749

[53] N.J. Cornish and J.J. Levin, Phys. Rev. Lett. 78 (1997) 998 
[54] J.D. Barrow and Y. Gaspar, Class. Quantum Grav. 18 (2001) 1809

[55] H. Ringström, Class. Quant. Grav. 18 (2001) 3791

[56] J. Wainwright, Gen. Rel. Grav. 32 (2000) 1041

[57] Y. Gaspar, Late time behaviour of general solutions of the Einstein field equations, PhD Thesis, Cambridge University (2001)

[58] E. Weber, J. Math. Phys. 28 (1987) 1658

[59] R.M. Wald, Phys. Rev. D28 (1983) 2118

[60] A.D. Linde and M.T. Zelnikov, Phys. Lett. B 215 (1988) 59

[61] J.D. Barrow and J. Yearsley, Class. Quantum Grav. 13 (1996) 2693

[62] J.D. Barrow, MNRAS 211 (1984) 221

[63] C.G. Hewitt, Gen. Rel. Grav 23 (1991) 691

[64] J. Wainwright, Gen. Rel. Grav. 16 (1984) 657

[65] O.I. Bogoyavlensky and S.P. Novikov, Sel. Math. Sov. 2 (1982) 159

[66] O.I. Bogoyavlensky, Methods in the Qualitative Theory of Dynamical Systems in Astrophysics and Gas Dynamics, Springer-Verlag (1985) 